\title{
Modelling and Repeating Early Photographic Positions with Aerial Drones
}

\author{
Michael E. Hodgson ${ }^{\mathrm{a}}$, Silvia E. Piovan ${ }^{\mathrm{b}}$ \\ a Department of Geography, University of South Carolina, Columbia, SC (USA), hodgsonm@sc.edu \\ ${ }^{b}$ Department of Historical and Geographic Sciences and the Ancient World, University of Padova, Padova (Italy), \\ silvia.piovan@unipd.it \\ * Corresponding author
}

Keywords: Repeat photography, drones, photogrammetry, modelling, cultural landscapes

\begin{abstract}
:
The use of repeat photography for documenting a changing landscape has a long history in geography. Repetitive photography has also been used in a photogrammetric context whereby changes in landscape and cultural features can be analytically measured and quantified. Such natural environment applications include changes (position, size, type) of forests, glaciers, landslides, or incised rivers. Similarly, the changes in anthropogenic features, such as buildings, bridges, roads, can also be quantified using photogrammetric principles.

One of the major challenges in acquiring current imagery for a repeat photography study of cultural landscapes is positioning the camera at the same, or nearly the same, location as the original photographer. For simple visualization of repeat photos the precision and accuracy required is modest. However, in the process of measuring features or changes in features matching the location of the camera and camera lens characteristics (e.g. angular field of view) must be very precise and appropriate for the application requirements. Determining the location of the early photo is not trivial as, in most instances, the optical center of the historic photo is not the center of the published photo. Similar to professional photographers today, earlier photos were often cropped and thus, the optical center is unknown.
\end{abstract}

Furthermore, the location of the earlier photographer may not have been on the ground or the ground location has changed. For example, many earlier photos were captured from the photographer on a scaffold or from a building. If the photographer was standing on the ground the surface may have since been modified through anthropogenic grading, natural erosion, or mass movements. For rural communities a common change in the landscape is the regrowth of vegetation, particularly forests, over the locations from which the historic photograph was taken. The issues of relocating the position of the early photographer are further complicated by access to property where the photographer was located.

The recent emergence of small unmanned aerial systems (sUAS) that are stable (e.g. multi-copters) and carry quality cameras has enabled almost any scientist or artist to collect landscape imagery "from above". With proper geospatial modelling the original geospatial position of the early photographer's camera can be estimated or located precisely with appropriate ancillary data. Additionally, the early photographer's lens angular field of view can also be determined. Finally, using sUAS contemporary photographs of the same landscape or environment can be collected from the same or nearly the same locations of the original photographer.

In this research we describe the salient characteristics required of the photographs, camera lens, and analytical modelling for determining the early photographer's location. A GIS-based modelling approach with both natural landscape and anthropogenic features in publically available databases are used to determine the photographer's location. Several case studies using the combined GIS-based model and sUAS are provided to demonstrate the feasibility of this novel approach.

One of the largest historic mining communities in the USA - Lynch, Kentucky - was well documented with early photography in the early 1900s, beginning in 1918 (shown in Figures 1, 2 and 3). Historic photographs were taken of the valley prior to development and during construction of the mining features, buildings, and residential housing. Unfortunately, many of these early photographs were taken from vantage points that are now forested over, making exact repositioning problematic. Ground positions of both historic and recent photographers are shown in Figure 1. However, modelling the location of these early positions was possible with a photogrammetric solution using LiDARderived topography, building footprints, and a sample of projected building facades. In the locations where forests completely occlude the former photographer's location we used an aerial drone camera to emulate nearly the identical location, albeit slightly away from the original position, such as in Figure 2 and 3. Using a selection of suburban sites in the Veneto region of Italy and the coal mining areas of Appalachia we demonstrate the relocation of early 
photographer's position using an analytical geospatial approach, at low altitude to moderate altitudes. This approach can be used for collecting repeat photography in other compromised places.
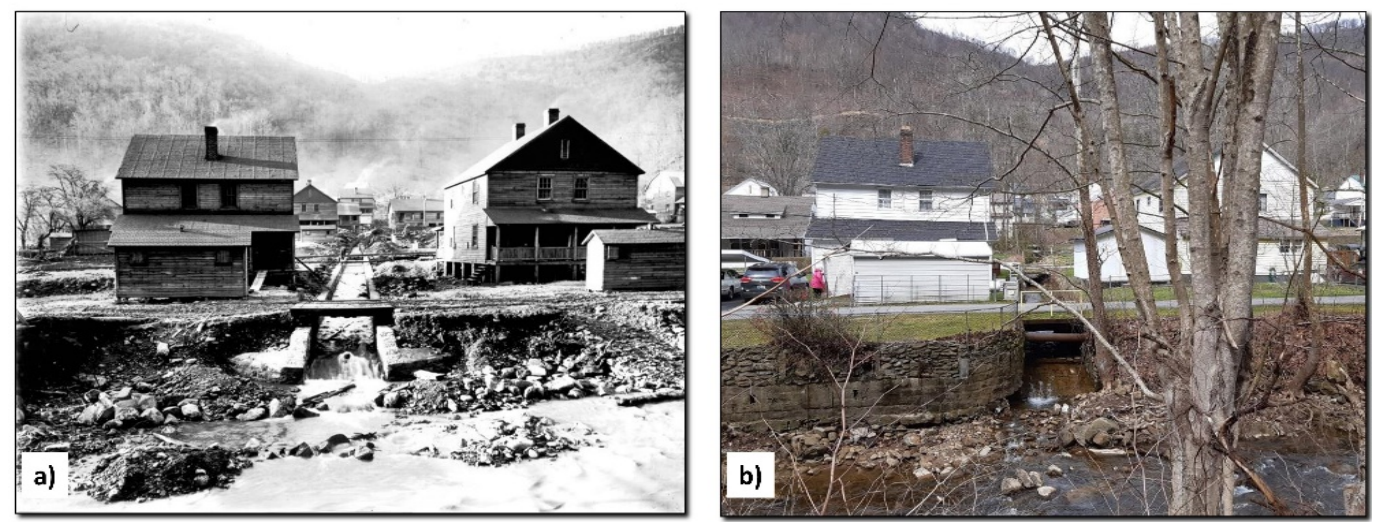

Figure 1. Historic photograph (1920) of a residential area and creek in Lynch, KY (a). In this instance, a repeat contemporary (2020) photograph was also taken from the ground although current vegetation somewhat obscures the scene.
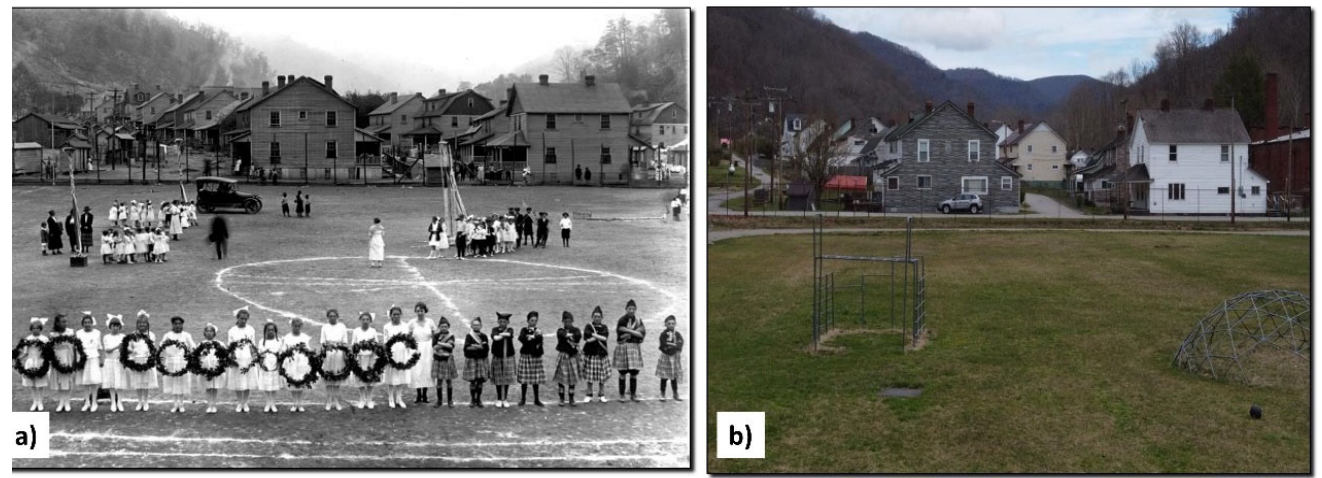

Figure 2. Historic photograph (1920) of the recreational field and adjacent residential area in Lynch, KY in a). Historic photographer was likely standing on temporary scaffold. Recent photo (2019) from low altitude aerial drone in b).
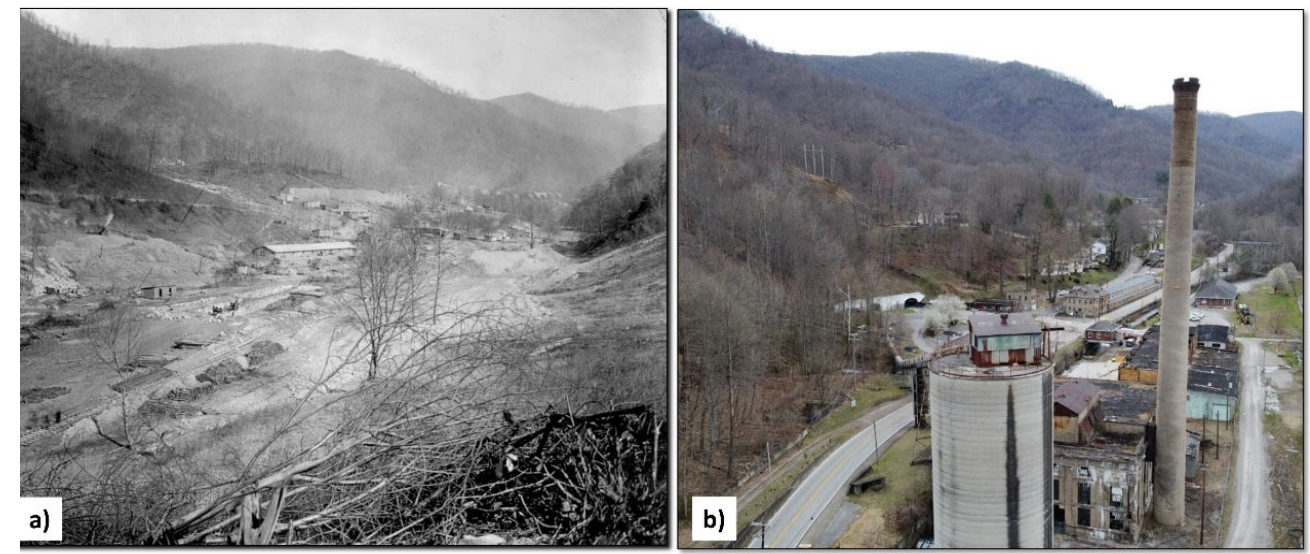

Figure 3. Historic photograph (1918) of the Lynch valley prior to coal milling building construction (a) where photographer was standing on a hill slope. Recent photo (2020) from moderate altitude aerial drone, slightly moved away from now tree-covered hillslope, shown in b). 